\title{
Robust Tuning of Power System Stabilizers in Multimachine Power Systems
}

\author{
Y. L. Abdel-Magid, Senior Member, IEEE, M. A. Abido, Member, IEEE, and A. H. Mantawy, Member, IEEE
}

\begin{abstract}
This paper demonstrates the robust tuning of power systems stabilizers for power systems, operating at different loading conditions. A classical lead-lag power system stabilizer is used to demonstrate the technique. The problem of selecting the stabilizer parameters is converted to a simple optimization problem with an eigenvalue-based objective function, which is solved by a tabu search algorithm. The objective function allows the selection of the stabilizer parameters to optimally place the closed-loop eigenvalues in the left-hand side of a vertical line in the complex $s$-plane. The effectiveness of the stabilizers tuned using the suggested technique, in enhancing the stability of power systems, is confirmed through eigenvalue analysis and simulation results.
\end{abstract}

Index Terms-Low frequency oscillation, Tabu search, Power system stability, Robust control.

\section{INTRODUCTION}

$\mathbf{T}$ HE application of tabu search (TS) algorithms has recently attracted the attention of researchers in the field of artificial intelligence. From the literature, it is clearly seen that tabu search algorithms can provide powerful tools for optimization [1]-[4]. Tabu search is an iterative improvement procedure whose main advantage is its ability to escape local optima. Tabu search is independent of the complexity of the objective function considered. It suffices to specify the objective function and to place finite bounds on the parameters.

The importance of increasing the stability boundaries of synchronous machines equipped with fast-acting static exciters is very well known. Several techniques have been used for the design of supplementary excitation controllers for this purpose [5]-[19].

It is well known that machine parameters change with loading, making the dynamic behavior of the machine quite different at different operating conditions [6]-[8]. Consequently, a set of power system stabilizer [PSS] parameters which stabilizes the system under a certain operating condition may no longer yield satisfactory results when there is a drastic change in the operating point.

In this paper, the power system, operating at various loading conditions is treated as a finite set of plants, and a robust PSS with parameters that can simultaneously improve the damping of this set of plants is determined off-line using a tabu search and an objective function based on the system eigenvalues. The

Manuscript received October 19, 1998; revised June 1, 1999. This work was supported by King Fahd University of Petroleum and Minerals.

The authors are with the Electrical Engineering Department, King Fahd University of Petroleum and Minerals, Dhahran 31261, Saudi Arabia (e-mail: \{amagid; mabido; amantawy\}@kfupm.edu.sa).

Publisher Item Identifier S 0885-8950(00)03812-8.
PSS designed in this manner will perform well under various loading conditions and stability of the system is guaranteed. By contrast, a PSS designed for a certain operating point will only perform optimally at the design loading condition.

The suggested eigenvalue-based objective function will optimally place the closed-loop eigenvalues of the power system in the left-hand side of a vertical line in the complex s-plane. A single machine infinite bus system, and a multimachine power system are used to demonstrate the suggested technique

The TS can easily incorporate most types of constraints and structures on the controller and often leads to the global optimum [1]. Moreover, the problem of simultaneous eigenvalue placement, whose analytic solution is yet unknown, is equally and easily solved using TS as shown in this work.

Simulation results and eigenvalue analysis are used throughout the paper to assess the effectiveness of the technique suggested in this work.

\section{TABU SEARCH}

Tabu Search was originally proposed as an optimization tool by Glover in 1977 [1]-[4]. It is a conceptually simple and an elegant iterative technique for finding good solutions to optimization problems. In general terms, TS is characterized by its ability to escape local optima by using a short-term memory of recent solutions called the tabu list. Moreover, tabu search permits backtracking to previous solutions by using the aspiration criteria.

Tabu search is an iterative improvement procedure in that it goes from some initial feasible trial solution to another by making moves. It makes several candidate moves and selects the move producing the best solution among all candidate moves for the current iteration. The set of admissible moves forms a candidate list. The best candidate solution becomes the current solution. It is possible that the tabu search may visit the same solution again in latter iterations. Thus, there is a possibility of cycling. Tabu restriction is a means to avoid such cycling by making the current solution tabu i.e., forbidden. Tabu restrictions are enforced by a tabu list. The tabu list is a list containing forbidden moves (solutions). It is forbidden to accept the same solution as long as it is in the tabu list. Tabu restrictions allow the search to go beyond the points of local optimality while still making the best possible move in each iteration.

The tabu list is initially empty, constructed in the first $z$ iterations ( $z$ : tabu list size) and updated in latter iterations. The tabu list ensures that the tabu search avoids returning to the solutions just visited and since degrading moves are allowed, the tabu search algorithm has a chance of leaving a local minimum [3]. 
Special attention must be given to the tabu list size. If the tabu list size is too small, the search process may start cycling and if it is too large, the search may be too restrictive. Therefore, an appropriate list size has to be determined by noting the occurrence of cycling when the size is too small and the quality of the solution when the size is too large [3].

The aspiration criterion is usually introduced to speed up the tabu search process. The aspiration criterion temporarily overrides the tabu status of a move if it is sufficiently good. The simplest aspiration criterion is to override the tabu status of a move and allow it into the tabu list if it leads to a better solution that the best obtained thus far [4].

Tabu search algorithms are more likely to converge to global optima than conventional optimization techniques and can also tolerate discontinuities, nonlinearities and noisy function evaluations.

\section{PROBlem Formulation AND RESUltS}

In this section, the eigenvalue-based objective function used to robustly select the PSS parameters is formulated, and the optimization problem solved with a tabu search algorithm. In a typical run of the TS, an initial solution is randomly generated. This initial solution is also assumed to be the current best solution. The current solution is perturbed with moves to generate a new set of trial solutions. Each move generates a trial solution. The number of moves to be made depends on the problem being considered. The move that generates the best solution among the set of trial solutions is selected. This move called the candidate move is checked to see if it is tabu. If it is not in the tabu list, it becomes the current solution. If the candidate solution is found to be tabu, its aspiration criterion is checked. If it passes the aspiration criterion, then it becomes the current best solution, otherwise moves are regenerated to get another set of new solutions and the process is repeated. The tabu search is terminated as soon as the stopping criteria are satisfied [3]. The tabu search algorithm is shown in Appendix II.

Consider the problem of determining the parameters of a stabilizer that relatively stabilizes a family of $N$ plants:

$$
\dot{X}(t)=A_{k} X(t)+B_{k} U(t) ; k=1,2, \ldots, N
$$

where $X(t) \in R^{n}$ is the state vector and $X(t) \in R^{m}$ is the control vector.

Very often, the closed-loop modes are specified to have some degree of relative stability. In this case, the closed-loop eigenvalues are constrained to lie to the left of a vertical line corresponding to a specified damping factor.

A necessary and sufficient condition for the set of plants in eqn. (1) to be simultaneously relatively stabilizable with a single control law is that the eigenvalues of the closed-loop system lie in the left-hand side of a vertical line in the complex $s$-plane [provided a stabilizing parameters set exists]. This condition motivates the following approach for determining the parameters of the PSS.

Select the parameters of the PSS to minimize the following objective function:

$$
J=\max \left\{\operatorname{Re}\left(\lambda_{k, i}\right)+\beta\right\} \quad k=1,2 \ldots, N ; i=1,2, \ldots, n
$$

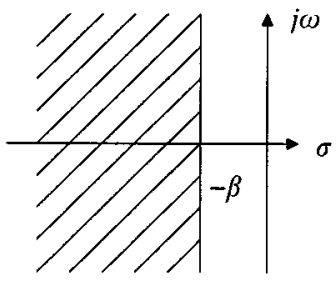

Fig. 1. Region in the left-hand side of a vertical line.

where $\lambda_{k, i}$ is the $i t h$ closed-loop eigenvalue of the $k t h$ plant, subject to the constraints that finite bounds are placed on the stabilizer parameters. The relative stability is determined by the value of $\beta$ as shown in Fig. 1. Plainly, if a solution is found such that $J<0$, then the resulting parameters simultaneously relatively-stabilize the collection of plants. The existence of a solution is verified numerically by minimizing $J$.

\section{SYSTEM MODEL}

In this study, two power systems are considered. The first system is a single-machine-infinite bus power system [9]. The second system is the 10-machine 39-bus New England power system [20], with each synchronous machine described by a nonlinear fourth-order model as given in the Appendix. The supplementary stabilizing signal considered is one proportional to speed. A widely used conventional PSS is considered throughout the study [9]. The transfer function of the ith PSS is:

$$
U_{i}(s)=K_{i} \frac{s \tau_{w i}}{1+s \tau_{w i}}\left[\frac{\left(1+s \tau_{1 i}\right)\left(1+s \tau_{3 i}\right)}{\left(1+s \tau_{2 i}\right)\left(1+s \tau_{4 i}\right)}\right] \Delta \omega_{i}(s)
$$

The structure of the PSS considered in (3) has been used by many researchers to assess and compare the results of their work. The first term in (3) is a washout term with a time lag $\tau_{w}$. The second term is a lead compensation to improve the phase lag through the system.

The numerical values of $\tau_{w}, \tau_{2}$ and $\tau_{4}$ used in the study are given in Appendix I.

The remaining parameters, namely, $K, \tau_{1}$, and $\tau_{3}$ are assumed to be adjustable parameters. The optimization problem, namely, the selection of these PSS parameters is easily and accurately solved using a tabu search. For a given operating point, the power system is linearized around the operating point, the eigenvalues of the closed-loop system are computed, and the objective function is evaluated. It is worth mentioning that only the system electromechanical modes are incorporated in the objective function. The bounds on the parameters used in the TS are given in Appendix I.

\section{Simulation Results}

To evaluate the effectiveness of the proposed technique, two power systems are studied.

\section{A. Example 1. Single-Machine-Infinite-Bus System}

In this part of the study, a single machine connected to infinite bus through a transmission line, and operating at different loading conditions, is considered. The linearized incremental 


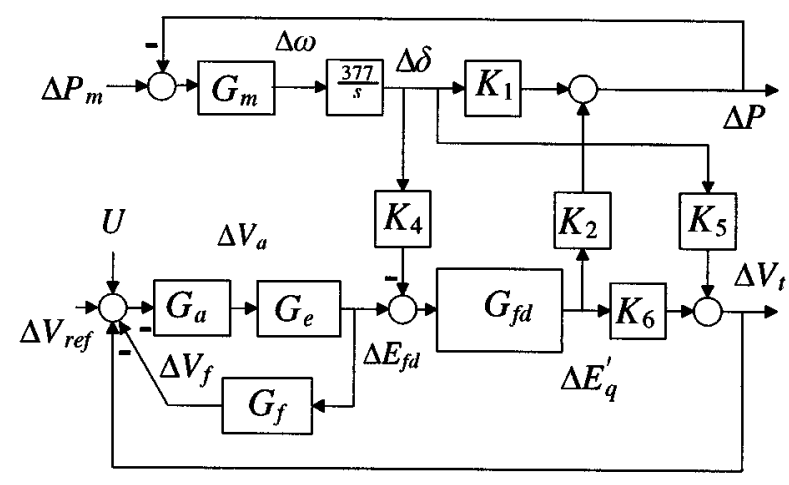

Fig. 2. System block diagram.

model of this system, voltage regulator and exciter included, is shown in Fig. 2.

The constants $K_{1}$ to $K_{6}$, with the exception of $K_{3}$, which is only a function of the ratio of the impedance, are dependent upon the actual real and reactive power loading as well as the excitation levels in the machine [7]. The system data are given in Appendix I.

The simultaneous damping enhancement of the system is demonstrated by considering five different loading conditions ( $N=5$ ). The operating points were selected randomly as follows:

$$
\begin{aligned}
\left(P_{o}, Q_{o}\right)= & (1.0,0.62) ;(1.0,0.2) ;(1.0,-0.1) \\
& (0.8,0.5) ;(1.0,1.0)
\end{aligned}
$$

The eigenvalues of the system at the five operating points considered, without PSS, are:

$$
\begin{aligned}
& -0.2350 \pm j 10.7853 ;-1.5520 ;-3.0830 \\
& -8.1340 \pm j 8.9851-0.2956 \pm j 11.5532 \\
& -1.7131 \pm j 0.8164 ;-8.6778 \pm j 9.1726 \\
& -0.2983 \pm j 12.1958 ;-1.3149 \pm j 1.0433 \\
& -9.0732 \pm j 9.4920-0.2818 \pm j 10.5746 \\
& -3.0260 ;-1.5411 ;-8.1210 \pm j 8.8397 \\
& -0.1463 \pm j 10.2375 ;-4.2045 \\
& -1.3478 ;-7.7639 \pm j 8.9775
\end{aligned}
$$

The damping ratios of the electromechanical modes are respectively:

\section{$[0.0218 ; 0.0256 ; 0.0244 ; 0.0266 ; 0.0143]$}

We used the objective function $J$ with the TS and $N=5$ to shift the electromechanical mode of each of the five systems to

\begin{tabular}{|c|c|}
\hline$[\mathrm{P}, \mathrm{Q}]$ & Eigenvalues \\
\hline$[1.0,0.62]$ & $\begin{array}{l}-\mathbf{3 . 0 7 9 1} \pm \mathbf{j} 13.3088 ;-46.0418 \pm \mathrm{j} 9.0617 ;-3.3298 \pm \mathrm{j} 8.4372 \\
-3.0253 ;-1.5518 ;-0.1\end{array}$ \\
\hline$[1.0,0.20]$ & $\begin{array}{l}-2.5750 \pm j 14.4546 ;-46.3536 \pm j 9.8142 ;-4.0932 \pm j 8.2693 \\
-1.7174 \pm j 0.8136 ;-0.1\end{array}$ \\
\hline$[1.0,-0.10]$ & $\begin{array}{l}-2.2614 \pm j ~ 15.3603 ;-46.5992 \pm j 10.3912 ;-4.5604 \pm j 8.3217 \\
-1.3182 \pm j 1.04752 ;-0.1\end{array}$ \\
\hline$[0.8,0.50]$ & $\begin{array}{l}-\mathbf{3 . 2 1 1 8} \pm \mathbf{j} 12.7591 ;-45.8969 \pm \mathrm{j} 8.6988 ;-3.3726 \pm \mathrm{j} 8.5365 \\
-2.9750 ;-1.5409 ;-0.1\end{array}$ \\
\hline$[1.0,1.0]$ & $\begin{array}{l}-3.6425 \pm \mathrm{j} 12.4684 ;-45.8129 \pm \mathrm{j} 8.4844 ;-2.6008 \pm \mathrm{j} 8.6655 \\
-4.0184 ;-1.3478 ;-0.1\end{array}$ \\
\hline
\end{tabular}
the left of the vertical line defined by $\beta=-2.2$ The gains of the PSS and $J$ in this case were found to be:

$$
K=0.4286, \tau_{1}=0.5665, \tau_{3}=0.7513 \text { and } J=-0.0614
$$

The eigenvalues of the five systems, with PSS, are given in Table I:

The damping ratios of the electromechanical modes are:

$$
[0.23 ; 0.178 ; 0.147 ; 0.292]
$$

TABLE I

EIGENVALUES WITH PSS [EXAMPLE 1]

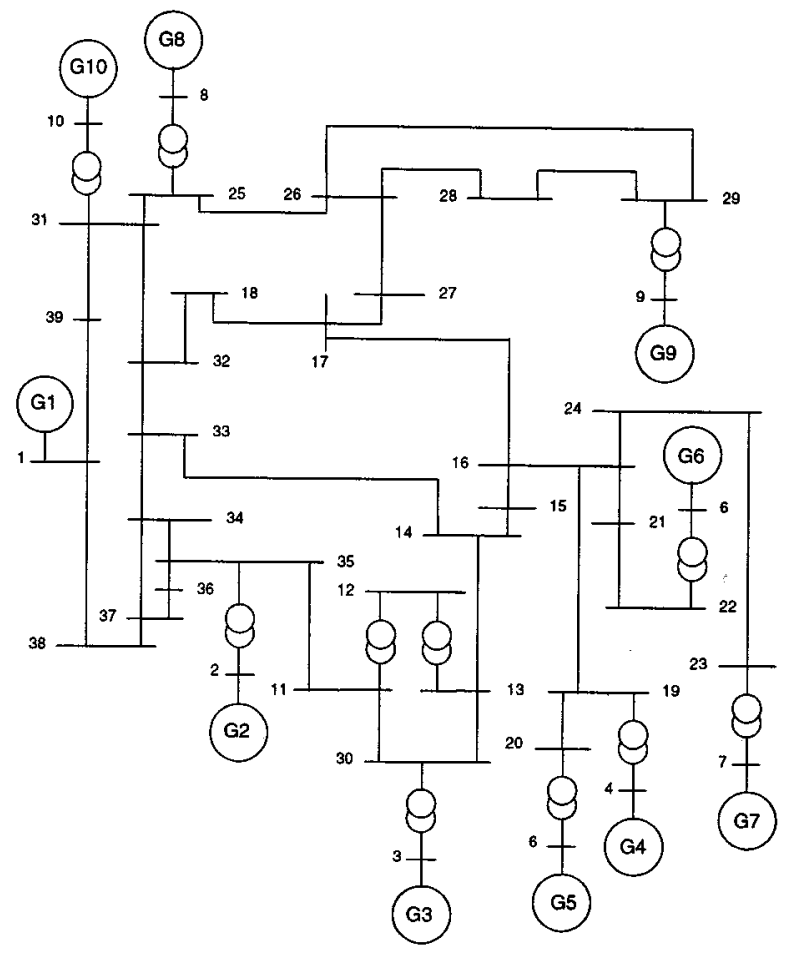

Fig. 3. Single line diagram for the New England System [20].

indicating a simultaneous improvement in the response of the five systems.

The extension of the suggested technique to the multimachine case is demonstrated next. In general, for a system consisting of $m$ machines each equipped with a stabilizer of the type described in this paper, the number of parameters to be optimized using the tabu search and objective function $J$ will be $3 \mathrm{~m}$, and the number of electromechanical modes to be shifted will be $m-1$. If $l$ operating points are selected for simultaneous stabilization, the $3 m$ parameters will be tuned such that the $l(m-1)$ modes are relocated as specified.

\section{B. Example 2. Thirty Nine-Bus Ten-Machine System}

In this part of the study, the 10-machine 39-bus power system shown in Fig. 3 is considered. Details of the system data are given in [20]. Generator $G_{1}$ is an equivalent power source representing parts of the U.S.-Canadian interconnection system. This 
TABLE II

EleCtromeChanical Modes; DAMPING Ratios [EXAMPLE 2, No PSSS]

\begin{tabular}{|c|c|c|}
\hline Nominal Loading & Light Loading & Heavy Loading \\
\hline $0.1497 \pm \mathrm{j} 9.7347 ; 0.0154$ & $0.1251 \pm \mathrm{j} 9.7589 ; 0.0128$ & $0.0474 \pm \mathrm{j} 9.9539 ; 0.0048$ \\
\hline $0.0974 \pm \mathrm{j} 9.7325 ; 0.0100$ & $0.1061 \pm \mathrm{j} 9.7251 ; 0.0109$ & $0.1271 \pm \mathrm{j} 9.9859 ; 0.0127$ \\
\hline $0.1596 \pm \mathrm{j} 9.3918 ; 0.0170$ & $0.1763 \pm \mathrm{j} 9.3650 ; 0.0188$ & $0.1874 \pm \mathrm{j} 9.6220 ; 0.0195$ \\
\hline $0.1060 \pm \mathrm{j} 8.2562 ; 0.0128$ & $0.0957 \pm \mathrm{j} 8.2862 ; 0.0115$ & $0.1652 \pm \mathrm{j} 8.2474 ; 0.0200$ \\
\hline $0.1602 \pm \mathrm{j} 8.0703 ; 0.0198$ & $-0.1133 \pm \mathrm{j} 8.0257 ; 0.0141$ & $0.0986 \pm \mathrm{j} 8.0706 ; 0.0122$ \\
\hline $0.1048 \pm \mathrm{j} 7.3998 ; 0.0142$ & $0.1036 \pm \mathrm{j} 7.4389 ; 0.0139$ & $0.0245 \pm \mathrm{j} 7.2248 ; 0.0034$ \\
\hline $0.1208 \pm \mathrm{j} 6.7662 ; 0.0179$ & $0.1267 \pm \mathrm{j} 6.8346 ; 0.0185$ & $0.1202 \pm \mathrm{j} 6.5599 ; 0.0183$ \\
\hline $0.0487 \pm \mathrm{j} 6.2368 ; 0.0078$ & $0.0908 \pm \mathrm{j} 6.3413 ; 0.0143$ & $0.0713 \pm \mathrm{j} \mathbf{6 . 0 8 2 8} ; \boldsymbol{- 0 . 0 1 1 7}$ \\
\hline $0.0344 \pm \mathrm{j} 4.3339 ; 0.0079$ & $0.0591 \pm \mathrm{j} 4.4094 ; 0.0134$ & $0.0240 \pm \mathrm{j} 4.2226 ; 0.0057$ \\
\hline
\end{tabular}

TABLE III

PSS PARAMETERS [EXAMPLE 2, WITH $J$ ]

\begin{tabular}{cccc}
\hline PSS on Generator & $K$ & $\tau_{1}$ & $\tau_{3}$ \\
\hline $\mathrm{G}_{5}$ & 12.258 & 0.3583 & 0.3465 \\
$\mathrm{G}_{7}$ & 10.981 & 0.6552 & 0.563 \\
$\mathrm{G}_{9}$ & 19.758 & 0.2033 & 0.06 \\
\hline
\end{tabular}

large system was selected to further demonstrate the versatility of the suggested technique.

Without power system stabilizers, the system damping is poor and the system exhibits highly oscillatory response [20]. It is therefore necessary to install one or more PSS to improve the dynamic performance. To identify the optimum locations of PSS's, the participation factor method [21] and the sensitivity of PSS effect method [22] were used. The results indicate that machines $G_{5}, G_{7}$ and $G_{9}$ are the optimum locations for installing PSS's to damp out the local and inter-areas modes of oscillations.

The robust tuning of the PSS's is demonstrated by considering three operating points labeled as nominal, light and heavy loading conditions. These loading conditions were obtained by varying the load admittances of the system.

The system nine electromechanical modes and the damping ratio without PSS's, for the three operating points considered, are given in Table II. Note that the system is unstable for heavy loading.

Using $J$ as the objective function with, $\beta=0.12$, the optimum values of the PSS parameters for the three PSS's used are given in Table III.

The objective function achieved is $J=-0.0058$ indicating that the mechanical modes have been shifted to the left of the vertical line $s=-0.12$ in the complex $s$-plane.

The system electromechanical modes and damping ratios, with the parameters of the PSS's set as in Table III, are given in Table IV.

The dynamic responses of the system to a 6-cycle three-phase fault near bus 29 at the end of line 26-29 are shown in Figs. 4-6, for the three loading conditions, and with parameters of the PSS's set as in Table III. The robustness of the PSS is quite
TABLE IV

Electromechanical Modes; Damping Ratios [EXAmple 2, with $J$ ]

\begin{tabular}{|c|c|c|}
\hline Nominal Loading & Light Loading & Heavy Loading \\
\hline $0.1508 \pm \mathrm{j} 9.7337 ; 0.0155$ & $0.126 \pm \mathrm{j} 9.7589 ; 0.0129$ & $0.1274 \pm \mathrm{j} 9.985 ; 0.0128$ \\
\hline $0.3238 \pm \mathrm{j} 9.1759 ; 0.0353$ & $-0.3376 \pm \mathrm{j} 9.1384 ; 0.0369$ & $0.5080 \pm \mathrm{j} 9.2567 ; 0.0548$ \\
\hline $0.2641 \pm \mathrm{j} 8.505 ; 0.0310$ & $0.2268 \pm \mathrm{j} 8.4961 ; 0.0267$ & $0.2569 \pm \mathrm{j} 8.3878 ; 0.0306$ \\
\hline $0.3593 \pm \mathrm{j} 8.0834 ; 0.0444$ & $0.3652 \pm \mathrm{j} 8.0889 ; 0.0451$ & $0.1828 \pm \mathrm{j} 8.2543 ; 0.0221$ \\
\hline $0.1707 \pm \mathrm{j} 8.0755 ; 0.0211$ & $0.1262 \pm \mathrm{j} 8.0333 ; 0.0157$ & $0.4300 \pm \mathrm{j} 8.1517 ; 0.0527$ \\
\hline $0.3042 \pm \mathrm{j} 7.3909 ; 0.0411$ & $0.2918 \pm \mathrm{j} 7.3869 ; 0.0395$ & $0.4083 \pm \mathrm{j} 7.2589 ; 0.0562$ \\
\hline $0.5129 \pm \mathrm{j} 5.6482 ; 0.0904$ & $0.5183 \pm \mathrm{j} 5.8046 ; 0.0889$ & $0.4178 \pm \mathrm{j} 5.9442 ; 0.0701$ \\
\hline $1.9494 \pm \mathrm{j} 3.5701 ; 0.4792$ & $-2.1757 \pm \mathrm{j} 3.7565 ; 0.5012$ & $1.4731 \pm \mathrm{j} 3.8001 ; 0.3614$ \\
\hline $0.2538 \pm \mathrm{j} 3.4892 ; 0.0725$ & $0.2749 \pm \mathrm{j} 3.7645 ; 0.0728$ & $0.2513 \pm \mathrm{j} 3.2082 ; 0.0781$ \\
\hline
\end{tabular}

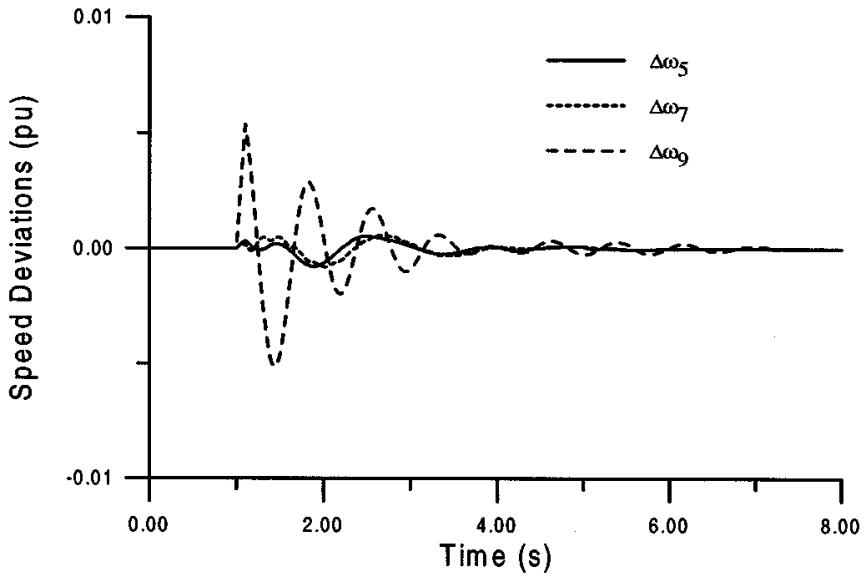

Fig. 4. Response to a three-phase fault with nominal conditions [New England system: $N=3$, objective function $J$ ].

evident. An excellent improvement in the damping over a wide range of operating conditions have been achieved with one set of PSS parameters.

\section{CONCLUSION}

The use of tabu search to design robust power system stabilizers for power systems working at various operating conditions is investigated in this paper. The problem of selecting the PSS parameters, which simultaneously improve the damping at various operating conditions, is converted to an optimization problem with an eigenvalue-based objective function which is solved by a tabu search algorithm. An objective function is presented allowing the robust selection of the stabilizer parameters that will optimally place the closed-loop eigenvalues in the left-hand side of a vertical line in the complex $s$-plane.

The suggested robust design technique was successfully demonstrated on a single-machine infinite bus system, and a multimachine power system, operating at different loading conditions.

The performance of the robust PSS's, tuned using the suggested technique, is verified through eigenvalue analysis and simulation results. 


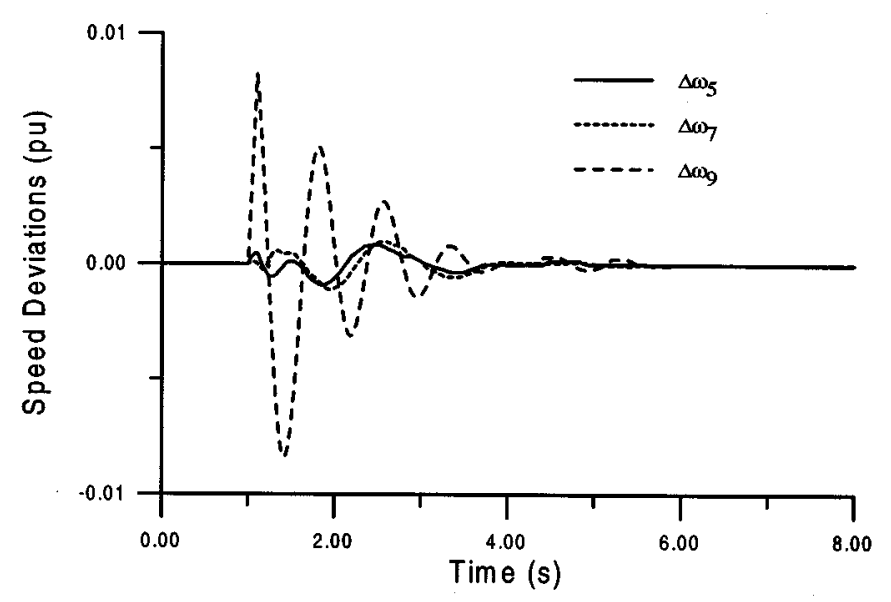

Fig. 5. Response to a three-phase fault with light conditions [New England system: $N=3$, objective function $J$ ].

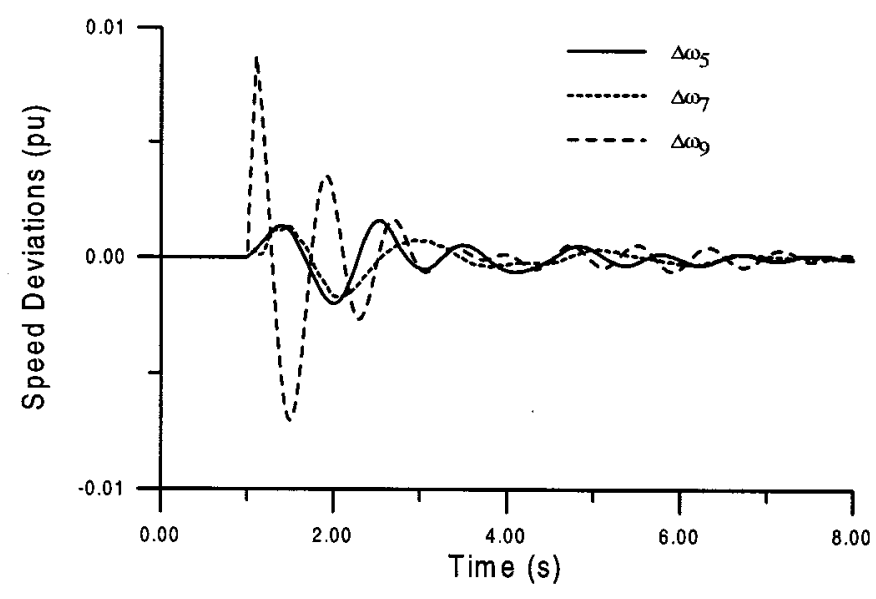

Fig. 6. Response to a three-phase fault with heavy conditions [New England system: $N=3$, objective function $J$ ].

\section{APPENDIX I \\ SYSTEM DATA}

Single-Machine-Infinite-Bus System

$$
\begin{aligned}
& G_{m}=\frac{1}{M s+D} ; G_{a}=\frac{K_{A}}{s T_{A}+1} ; G_{e}=\frac{1}{s T_{E}+K_{E}} \\
& G_{f}=\frac{s K_{F}}{1+s T_{F}} ; G_{f d}=\frac{K_{3}}{1+s T_{d 0}^{\prime} K_{3}}
\end{aligned}
$$

The system data are as follows [9]:

Machine (p.u.)

$$
\begin{aligned}
& x_{d}=1.7 \quad ; \quad x_{d}^{\prime}=0.254 \quad ; \quad x_{q}=1.64 \\
& \omega_{0}=120 \pi \mathrm{rad} / \mathrm{s} \quad ; \quad T_{d 0}^{\prime}=5.9 \mathrm{sec} \\
& D=0.0 ; \quad M=4.74 \mathrm{sec}
\end{aligned}
$$

Transmission Line (p.u.)

$$
r_{e}=0.02 ; x_{e}=0.4
$$

\section{Exciter and Stabilizer}

$$
\begin{aligned}
& K_{A}=400 ; T_{A}=0.05 \mathrm{sec} ; K_{F}=0.025 ; T_{F}=1.0 \mathrm{sec} \\
& K_{E}=-0.17 ; T_{E}=0.95 \mathrm{sec} \\
& \tau_{w}=10 \mathrm{sec} ; \tau_{2}=\tau_{4}=0.0227 \mathrm{sec} .
\end{aligned}
$$

bounds for the stabilizer adjustable gain and time constants are $[0.01,10]$ and $[0.03,1.0]$, respectively.

\section{Multimachine Power System [20]}

Machine Model:

$$
\begin{gathered}
\dot{\delta}_{i}=\omega_{b}\left(\omega_{i}-1\right) \\
\dot{\omega}_{i}=\frac{T_{m i}-T_{e i}-D_{i}\left(\omega_{i}-1\right)}{M_{i}} \\
{\stackrel{\bullet}{E_{q i}}}^{\prime}=\frac{E_{f d i}-\left(x_{d i}-x_{d i}^{\prime}\right) i_{d i}-E_{q i}^{\prime}}{T_{d o i}^{\prime}} \\
\dot{\bullet}_{f d i}=\frac{K_{a i}\left(V_{r e f i}-V_{i}+U_{i}\right)-E_{f d i}}{T_{a i}} \\
T_{e i}=E_{q i}^{\prime} i_{q i}-\left(x_{q i}^{\prime}-x_{d i}^{\prime}\right) i_{d i} i_{q i}
\end{gathered}
$$

$$
\tau_{w}=10 \mathrm{sec} \quad ; \quad \tau_{2}=\tau_{4}=0.05 \mathrm{sec} .
$$

bounds for the stabilizer adjustable gain and time constants are $[0.01,20]$ and $[0.06,0.8]$, respectively.

\section{APPENDIX II \\ TABU SEARCh AlgORITHM}

The following notation is used in the description of the tabu search algorithm:

$X: \quad$ The set of feasible solutions for a given problem.

$x: \quad$ Current solution, $x \in X$

$x^{\prime \prime}: \quad$ Best solution reached

$x^{\prime}$ : $\quad$ Best solution among a sample of trial solutions.

$E(x): \quad$ Evaluation function of solution $x$

$N(x)$ : Set of neighborhood of $x \in X$ (trial solutions)

$S(x): \quad$ Sample of neighborhood of $x ; S(x) \in N(x)$

$S S(x)$ : Sorted sample in ascending order according to their evaluation functions, $E(x)$

TL: $\quad$ Tabu list.

AL: $\quad$ Aspiration level.

Step(0): Set tabu list (TL) as empty and aspiration level (AL) to be zero. 
Step(1): Set iteration counter $K=0$. Select an initial solution $x \in X$, and set $x^{\prime \prime}=x$.

Step(2): Generate randomly a set of trial solutions $S(x) \in$ $N(x)$ (neighbor to the current solution $x$ ) and sort them in an ascending order, to obtain $S S(x)$. Let $x^{\prime}$ be the best trial solution in the sorted set $S S(x)$ (the first in the sorted set).

Step(3): If $E\left(x^{\prime}\right)>E\left(x^{\prime \prime}\right)$, go to Step(4), else set the best solution $x^{\prime \prime}=x^{\prime}$ and go to $\operatorname{Step}(4)$.

Step(4): Perform the tabu test. If $x^{\prime}$ is NOT in the TL, then accept it as a current solution, set $x=x^{\prime}$, and update the TL and AL and go to Step(7), else go to Step(5).

Step(5): Perform the AL test. If satisfied, then override the tabu state, Set $x=x^{\prime}$, update the AL and go to $\operatorname{Step}(7)$, else go to Step(6).

Step(6): If the end of the $S S(x)$ is reached, go to $\operatorname{Step}(7)$, otherwise, let $x^{\prime}$ be the next solution in the $S S(x)$ and go to $\operatorname{Step}(4)$.

Step(7): Perform termination test. If the stopping criterion is satisfied then stop, else Set $K=K+1$ and go to Step (2).

In our implementation, the search is stopped if either of the following two conditions is satisfied:

- The number of iterations performed since the best solution last changed is greater than a prespecified maximum number of iterations; or

- Maximum allowable number of iterations is reached.

\section{REFERENCES}

[1] F. Glover and H. J. Greenberg, "New approaches for heuristic search: a bilateral linkage with artificial intelligence," European Journal of Operational Research, vol. 39, no. 2, pp. 119-130, 1989.

[2] F. Glover, "Artificial intelligence, heuristic frameworks and tabu search," Managerial and Decision Economics, vol. 11, pp. 365-375, 1990.

[3] F. Glover, "Tabu search: part 1," ORSA Journal on Computing, vol. 1, no. 3, pp. 190-206, summer 1989.

[4] F. Glover, "A user's guide to tabu search," Annals of Oper. Reas., vol. 41 , pp. 3-28, 1993.

[5] F.R. Scheif, H.D. Hunkins, G.E. Martin,, and E.E. Hattan, "Excitation control to improve power line stability," IEEE Transactions on Power Apparatus and Systems, vol. PAS-87, pp. 1426-1434, 1968.

[6] F.P. de Mello and C. Concordia, "Concepts of synchronous machine stability as affected by excitation control," IEEE Transactions on Power Appparatus and Systems, vol. PAS-88, pp. 316-327, 1969.

[7] M. K. El-Sherbini and D. M. Mehta, "Dynamic Systems stability - investigation of the effect of different loadings and excitation systems," IEEE Transactions on Power Appparatus and Systems, vol. PAS-92, pp. 1538-1546, 1973.

[8] Y. L. Abdel-Magid and G. W. Swift, "Variable structure power stabilizer to supplement static-excitation system," PROC. IEE, vol. 123, no. 7, pp. 697-701, July 1976.

[9] P.M. Anderson and A.A. Fouad, Power System Control and Stability. Iowa: Iowa State University Press, 1977.

[10] Y.N. Yu, Electric Power System Dynamics. New York: Academic Press, 1983

[11] H. A. M. Moussa and Y.N. Yu, "Optimal power system stabilization through excitation and/or governor control," IEEE Transactions on Power Appparatus and Systems, vol. PAS-91, pp. 1166-1174, 1972.

[12] W.C. Chan and Y.Y. Hsu, "An optimal variable structure power system stabilizer for power stabilization," IEEE Transactions on Power Appparatus and Systems, vol. PAS-102, pp. 1738-1746, 1983.

[13] Y.Y. Hsu and C. Y. Hsu, "Design of a proportional-integral power system stabilizer," IEEE Transactions on Power Systems, vol. PWRS-1, no. 2, pp. 46-83, 1986
[14] A. Feliachi and X. Zhang et al., "Power system stabilizers design using Optimal reduced order models, Part I: Model Reduction," IEEE Transactions on Power Systems, vol. PWRS-3, no. 4, pp. 1670-1675, 1988.

[15] E.V. Larsen and D.A. Swann, "Applying Power System Stabilizers: Parts I, II, and III," IEEE Transactions on Power Apparatus and Systems, vol. PAS-100, pp. 3017-3046, 1981.

[16] P. Kundur, M. Klein, G.J. Rogers, and M.S. Zwyno, "Application of power system stabilizers for enhancement of overall system stability," IEEE Transactions on Power Systems, vol. 4, pp. 382-388, September 1992

[17] P. Pourbeik and M.I. Gibbard, "Simultaneous coordination of power system stabilizers and FACTS device stabilizers in a Multi-Machine power system for enhancing dynamic performance," IEEE Transactions on Power Systems, vol. 13, pp. 473-479, 1998.

[18] Y. Abdel-Magid, M. Bettayeb, and M.M. Dawoud, "Simultaneous stabilization of power systems using genetic algorithms," IEE Proc.-Gener. Transm. Distrib., vol. 144, no. 1, pp. 39-44, January 1997.

[19] G.N. Taranto and D.M. Falcao, "Robust decentralized control design using genetic algorithms in power system damping control," IEE Proc.Gener. Transm. Distrib., vol. 145, no. 1, pp. 1-6, January 1998.

[20] T. Hiyama and T. Sameshima, "Fuzzy logic control scheme for on-line stabilization of multimachine power system," Fuzzy Sets and Systems, vol. 39, pp. 181-194, 1991.

[21] Y. Y. Hsu and C. L. Chen, "Identification of optimum location for stabilizer applications using participation factors," in IEE Proceedings, Pt. C, vol. 134, May 1987, pp. 238-244.

[22] E. Z. Zhou, O. P. Malik, and G. S. Hope, "Theory and method for selection of power system stabilizer location," IEEE Trans. on Energy Conversion, vol. 6, no. 1, pp. 170-176, 1991.

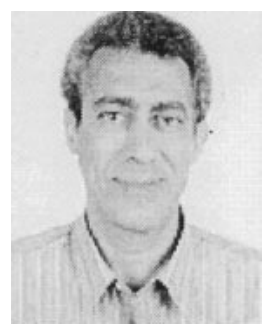

Y. L. Abdel-Magid (M'74, SM'87) received the B. Sc. (Honors) from Cairo University, Egypt, in 1969 and the M. Sc. and the Ph. D. degrees from the University of Manitoba, Winnipeg, Canada, in 1972 and 1976 respectively, all in Electrical Engineering. From 1976 to 1979, he was with Manitoba Hydro as a Telecontrol engineer. During the 1990-1991 academic year, he was a visiting scholar at Stanford University, Palo Alto, CA, USA. His research interests include power system control and operation, adaptive and robust control of power systems, and applications of AI techniques in power systems.

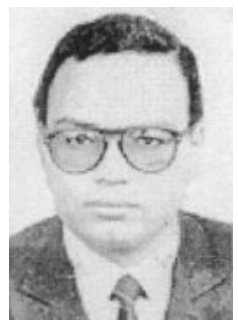

M. A. Abido received the B.Sc. (Honors ) and the M.Sc. degrees in Electrical Engineering from Menofia University, Egypt, in 1985 and 1989 respectively, and the Ph. D. degree from King Fahd University of Petroleum and Minerals, Saudi Arabia, in 1997. His research interests are system identification, power system control and applications of AI techniques in power systems.

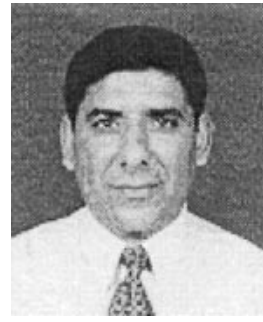

A. H. Mantawy received the B.Sc. (Honors) and the M.Sc. degrees in Electrical Engineering from Ain Shams University, Egypt, in 1982 and 1988 respectively, and the $\mathrm{Ph}$. D. degree from King Fahd University of Petroleum and Minerals, Saudi Arabia, in 1997. His research interests includes the applications of AI techniques to power system operation and planning. 